A N N A L E S Annales de Bretagne et des Pays de l'Ouest

Anjou. Maine. Poitou-Charente. Touraine

114-4 | 2007

Varia

\title{
La bataille de Saint-Cast (1758) et sa mémoire : une mythologie bretonne
}

David Hopkin, Yann Lagadec et Stéphane Perréon

\section{(2) OpenEdition}

Journals

Édition électronique

URL : http://journals.openedition.org/abpo/480

DOI : $10.4000 / a b p o .480$

ISBN : 978-2-7535-1508-6

ISSN : 2108-6443

Éditeur

Presses universitaires de Rennes

Édition imprimée

Date de publication : 30 décembre 2007

Pagination : 195-215

ISBN : 978-2-7535-0598-8

ISSN : 0399-0826

\section{Référence électronique}

David Hopkin, Yann Lagadec et Stéphane Perréon, « La bataille de Saint-Cast (1758) et sa mémoire

une mythologie bretonne », Annales de Bretagne et des Pays de l'Ouest [En ligne], 114-4 | 2007, mis en ligne le 30 décembre 2009, consulté le 19 avril 2019. URL : http://journals.openedition.org/abpo/480 DOI : $10.4000 / a b p o .480$ 


\title{
La bataille de Saint-Cast (1758) et sa mémoire : une mythologie bretonne ${ }^{1}$
}

\author{
David HOPKIN \\ Fellow in Modern History, Hertford College, \\ Oxford University \\ Yann LAGADEC \\ Maître de conférences en histoire moderne, \\ CERHIO - Université Rennes 2 \\ Stéphane PERRÉON \\ Professeur agrégé au Lycée de Bressuire, docteur en histoire, \\ CERHIO - Université Rennes 2
}

L'ampleur prise à l'automne 1758 par les célébrations de la - militairement très modeste - victoire de Saint-Cast remportée le 11 septembre de la même année par les troupes du duc d'Aiguillon face à des soldats britanniques en cours de rembarquement a de quoi surprendre. Une dizaine de mois après la désastreuse défaite de Rossbach, quelques semaines après les raids destructeurs contre Cancale et Cherbourg, la retraite des forces du général Bligh - qui n'ont pu cette fois-ci approcher de Saint-Malo - vient certes à point nommé pour rassurer une opinion inquiète des évolutions du conflit tant dans le Saint-Empire qu'Outre-mer. Ce soulagement n'explique sans doute pourtant pas tout, et notamment le formidable engouement qui marque les derniers mois de 1758.

Ainsi le recteur de la petite paroisse de Saint-Cast est-il rapidement - et durablement, des semaines durant - submergé de "touristes " souhaitant visiter le champ de bataille, lieu de tous les exploits ${ }^{2}$. Un récit du combat victorieux, publié à Paris dès le 15 septembre 1758, est distribué gratuitement par des colporteurs, " ce qui ne s'étoit point encore vu à Paris " écrit

1. Cette recherche a bénéficié du soutien financier de la British Academy et de suggestions d'Éva Guillorel et Didier Guyvarc'h.

2. "Récit du Recteur de Saint-Cast ", dans Saint-Cast. Recueil de pièces officielles et de documents contemporains relatifs au combat du 11 septembre 1758, Saint-Brieuc, Prudhomme, 1858, p. 162. 
un témoin ${ }^{3}$. Louis XV ordonne qu'un Te Deum soit célébré à Notre-Dame le $1^{\mathrm{er}}$ octobre, une cérémonie que termine un feu d'artifices tiré devant l'Hôtelde-Ville ${ }^{4}$. À cette occasion, les maisons sont illuminées, de la nourriture est distribuée gratuitement, musique et danses animant la nuit parisienne jusqu'au petit matin ${ }^{5}$. Dans les semaines qui suivent, ces scènes à la fois religieuses et festives se répètent partout en France, et plus particulièrement en Bretagne ${ }^{6}$. Cartes, gravures, libelles sont diffusés par des imprimeurs de Rennes, Nantes ou Paris. En décembre, les états de Bretagne commandent au peintre et dessinateur Ozanne une gravure dont un exemplaire est offert à chacun des membres de l'assemblée provinciale mais aussi à chaque communauté de la province. Une médaille est même frappée en l'honneur du duc d'Aiguillon, bien que l'on décide qu'elle ne le sera qu'en "bronze seulement [...] attendu les misères de la province et le peu de fonds qu'elle $\mathrm{a}^{7}$ ". Chanteurs des rues et compositeurs de la cour célèbrent eux aussi, de concert, le commandant en chef victorieux qui, pour sa part, multiplie dans les mois qui suivent de véritables " entrées ducales " d'un bout à l'autre de la province. De manière plus anecdotique mais tout aussi révélatrice, une contredanse dite de Saint-Cast, " dont la première figure représente un exercice militaire ", voit le jour ${ }^{8}$. Ainsi que le note l'historien B. Pocquet, la bataille donna en 1758 le ton à la mode : " On porta des robes et des éventails à la Saint-Cast ${ }^{9}$."

La célébration de la victoire ne prend pourtant pas fin avec les durs revers de l'année suivante, non plus qu'en 1761 alors que les forces britanniques investissent Belle-Île ou en 1763 à l'annonce des clauses particulièrement défavorables du traité de Paris. Au contraire même. Après l'euphorie - souvent profane - de l'automne 1758, la religion se ressaisit de l'événement, permettant de voir s'opérer le basculement de la célébration à la commémoration. Plus que les nombreuses cérémonies visant à honorer les morts des combats, sans doute est-ce, en la matière, la procession parcourant chaque 11 septembre le champ de bataille qui apparaît comme la plus significative : on y remercie notamment Dieu d'avoir épargné le village

3. Coll., La Bretagne sous Louis XV. Exposition commémorative du combat de Saint-Cast (11 septembre 1758), Saint-Brieuc, Arch. dép. des Côtes-du-Nord, 1958, p. 2.

4. Ces "cérémonies de l'information" ne sont cependant pas étudiées par FogEL, Michèle, Les Cérémonies de l'information dans la France du XVe au XVIII siècle, Paris, Fayard, 1989 qui s'intéresse plus particulièrement au cas de la guerre de Succession d'Autriche.

5. " Lettre de Louis XV ", dans Saint-Cast. Recueil..., op. cit., p. 66.

6. Ainsi à Saint-Malo le 8 octobre, ou le 15 à Nantes. La Bretagne n'est cependant pas la seule province concernée : un mandement de l'évêque de Valence en date du 4 octobre 1758 demande ainsi qu'un Te Deum soit célébré dans son diocèse; ConDAN, "Bataille de Saint-Cast ", Revue de Bretagne, de Vendée et d'Anjou, Janvier, 1900, p. 43-46.

7. "États de Bretagne ", dans Saint-Cast. Recueil..., op. cit., p. 41-42.

8. «Extrait du registre du bureau de la communauté de Rennes " et " Extrait du registre du bureau de la communauté de Nantes ", dans : Saint-Cast. Recueil..., op. cit., p. 76 et 83. Voir aussi PoCQUET, Barthélemy, "La Chalotais et le parlement de Bretagne ", Bulletins et mémoires de la Société d'émulation des Côtes-du-Nord, 1895, p. 25.

9. Pocquet, Barthélemy, Histoire de Bretagne, Rennes, Plihon et Hommay, 1914, T. VI, p. 273. 
de Saint-Cast, alors que ceux de Saint-Briac et du Guildo ont été « réduits en cendres ${ }^{10}$ ". C'est dans ce contexte d'ailleurs qu'apparaissent toute une série de légendes, concernant pour certaines une statue de la Vierge de Pléboule, pour d'autres celle de Sainte-Blanche conservée dans une chapelle de pêcheurs à proximité du port de Saint-Cast ${ }^{11}$.

Pourtant, au cours du XIX ${ }^{\mathrm{e}}$ siècle, ces commémorations de nature religieuse cèdent le pas. Historiens et érudits s'emparent en effet de l'événement, participant à la construction d'une mémoire provinciale - pour ne pas dire régionaliste, voire nationaliste - de la bataille, une mémoire peu, voire pas étudiée jusqu'à ce jour ${ }^{12}$. L'espèce de " mythologie " régionale/nationale qui s'édifie alors s'articule autour de trois épisodes dont le processus d'élaboration nous retiendra plus particulièrement ici : l'appropriation bretonne de l'événement "Saint-Cast ", la disqualification du héros initial, allochtone, enfin l'effort en vue de la construction d'un nouveau héros, autochtone celui-là.

\section{L'appropriation bretonne de l'événement}

Très tôt dans le XIX ${ }^{\mathrm{e}}$ siècle, les élites littéraires et politiques bretonnes accordent à la bataille de Saint-Cast une importance sans commune mesure avec ses répercussions réelles sur le déroulement de la guerre de Sept Ans. L'assertion de Sigismond Ropartz dans son introduction au recueil de documents publié en 1858, selon laquelle " la bataille de Saint-Cast compte parmi les plus glorieux faits d'armes que l'histoire ait enregistrés " ne repose sur aucune réalité objective ${ }^{13}$. C'est pourtant ce que vont s'attacher à mettre en avant érudits et historiens bretons, entretenant ainsi une anglophobie bien ancrée tout en construisant progressivement le mythe d'une victoire de la Bretagne.

\section{L'affirmation d'une profonde anglophobie}

Comme a pu le faire remarquer J. Guiffan, « il n’y a pas moins de vingt articles dans les différentes revues bretonnes sur les attaques des Anglais contre les côtes bretonnes ${ }^{14}$ ". S'ils sont en fait bien plus nombreux - entre

10. "Récit du Recteur de Saint-Cast", dans : Saint-Cast. Recueil..., op. cit., p. 16. Supprimée après 1790, cette procession annuelle reprend après la Révolution; " Relation de la Bataille de Saint-Cast ", dans Le Lycée armoricain, 1823, p. 323.

11. SÉBILlOT, Paul, Traditions et superstitions de la Haute-Bretagne, Paris, Maisonneuve et Larose, 1882, vol. 1, p. 323 et 368.

12. L'ouvrage fondateur de GUIOMAR, Jean-Yves, Le Bretonisme. Les historiens bretons au XIX siècle, Rennes, Société d'histoire et d'archéologie de Bretagne, 1987, ne fait, par exemple, qu'effleurer le sujet, se concentrant sur les mythes des origines, celtiques ou médiévales, de la Bretagne, au cœur des préoccupations de la plupart des érudits du temps.

13. Ropartz, Sigismond, "Préface ", dans : Saint-Cast. Recueil..., op. cit., p. v; voir aussi La Noue, Frédéric de, Notice sur le combat de Saint-Cast (11 septembre 1758), Dinan, J. Bazouge, 1858, p. 95.

14. GuIfFAn, Jean, Histoire de l'anglophobie en France, Rennes, Terre de Brume, 2004, p. 214. 
80 et 100 , pour la plupart publiés entre 1820 et 1914 -, ces articles participent d'une anglophobie latente aux sources multiples. Deux éléments jouent notamment ici : d'une part, la concurrence halieutique du moment, d'autre part le souvenir - souvent vague - des rivalités maritimes passées, alimenté d'ailleurs par des tensions plus contemporaines, coloniales notamment.

Passons rapidement sur le premier de ces éléments ${ }^{15}$. Il ne joue en effet sans doute que dans des cercles limités, ceux du monde des gens de mer, confrontés très directement à l'usage de facto partagé du French Shore de Terre-neuve depuis 1713 et le traité d'Utrecht; des cercles limités à l'échelle du pays et même de la Bretagne, mais dont l'importance est bien plus considérable dans les zones littorales dévastées par les raids britanniques de 1758. Or ces tensions, héritées du XVIII ${ }^{\mathrm{e}}$ siècle, rejouent régulièrement au XIX ${ }^{\mathrm{e}}$ siècle, notamment en 1856 alors que Terre-neuve accède à une certaine autonomie, justifiant une intense campagne de presse en 1858, au moment du voyage en Bretagne de Napoléon III, au moment aussi du centenaire de la bataille de Saint-Cast. Ces tensions perdurent d'ailleurs jusqu'à l'abandon de cette zone de pêche, en 1904, dans le cadre des négociations devant conduire à la signature de l'Entente cordiale.

En effet, loin de soulager les discordes anciennes, le temps passant tend au contraire, notamment chez les hommes de lettres, à raviver une mémoire à peine enfouie. La chose est nette en 1858, au moment du centenaire de la bataille. Dans un article de la Foi bretonne, repris par la Revue de Bretagne et de Vendée en février 1858, A. de La Noue propose ainsi d'ériger à proximité de la plage " un gigantesque obélisque, si haut, si haut, que, sans approcher de la côte, les voiles anglaises sillonnant l'Océan puissent l'apercevoir et y lire, en gros et ineffaçables caractères, ces mots : On ne passe pas ${ }^{16}$ ! " En 1923 encore, le chanoine Lemasson, rééditant trois récits de contemporains des événements, n'hésite pas, à chaque page et parfois à chaque ligne, à entrecouper de "corrections " une narration trop tiède, à multiplier les références aux pillages et aux meurtres dus à la soldatesque ennemie ${ }^{17}$. La tendance à surestimer les pertes britanniques - et par là même, à enfler l'importance de la victoire française - relève de la même logique : selon Duclos par exemple, que l'on cite parfois complaisamment, le nombre de morts serait de 20000, un chiffre trois fois supérieur à celui des troupes débarquées ${ }^{18}$. Et le temps ne change rien à l'affaire. Ainsi est-ce dans un ouvrage bien plus récent, tenant certes plus de la brochure touristique locale que du livre d'histoire, que l'on trouve la description la plus violente

15. Sur ce point, Hopkin, David, Lagadec, Yann et Perreon, Stéphane, " The Experience and Culture of War in the Eighteenth-Century: The British Raids on the Breton Coast, 1758 ", French Historical Studies, 2008, à paraître.

16. Revue de Bretagne et de Vendée, 1858, février, p. 191.

17. Lemasson, Auguste, La Descente des Anglais à Saint-Briac et leur défaite à Saint-Cast l'an 1758, Saint-Brieuc, Guyon, 1923.

18. Saint-Cast. Recueil..., op. cit., p. 228. Les auteurs du recueil notent eux-mêmes que "Duclos exagère évidemment, dans ce passage, le nombre de nos ennemis ". 
de la bataille, une description parfois caricaturale d'ailleurs : à en croire les auteurs, " la vue du sang excite [les soldats français], plus ils tuent plus ils ont envie de tuer, rien ne les arrête, rien ne leur fait plus peur : ce sont des animaux assoiffés de chair humaine [...]. Le sang devient une obsession, un besoin, une drogue ${ }^{19}$ ". L'explication de ce déchaînement serait à chercher, selon A. Oléron et E. Rondel, dans la volonté de se venger des incendies, maraudages, sacrilèges envers les biens d'Eglise et les membres du clergé dus aux troupes de Bligh durant la semaine que dura leur raid.

Le pillage et le maraudage seraient ainsi, selon nombre d'écrivains bretons des $\mathrm{XIX}^{\mathrm{e}}$ et $\mathrm{XX}^{\mathrm{e}}$ siècles, comme des vices anglais de nature quasi " raciale ", vices dont les expéditions de 1758 - celle de Saint-Cast en septembre comme celle de Cancale en juin - ne seraient qu'une énième illustration. Ces traits de caractère durables des Anglais - et, au-delà, des Britanniques -, s'inscriraient dans le temps long des déprédations, récurrentes depuis la conquête anglo-saxonne de la Bretagne - la Grande... - au V $\mathrm{V}^{\mathrm{e}}$ siècle ${ }^{20}$. S'il est bien évidemment difficile de trouver des références contemporaines à une haine héréditaire que voueraient les Bretons aux Saxons, elles ne sont pas rares dans les récits des deux derniers siècles. "Breiz ha Bro-zaoz enebourien/Evit-ho bout amezeien/A zo bet laket er bedmen/D'en emfibla da virviken ${ }^{21}$ ": c'est ainsi, par exemple, que la présente La Villemarqué dans son Emgann Sant-Cast. La bataille de 1758 s'inscrirait donc dans cette continuité, renforçant la prégnance de l'événement dans l'esprit des Bretons, l'anglophobie latente n'en rendant que plus facile l'érection de l'épisode en une sorte de « lieu de mémoire » breton.

\section{Un « lieu de mémoire » pour tous les Bretons}

La présence d'Emgann Sant-Cast parmi les textes du Barzaz-Breiz, ouvrage dont la publication est considérée par certains comme l'un des moments fondateurs du mouvement breton moderne, dit bien le statut que l'on cherche à assigner à la bataille dans la mémoire régionale. S'inspirant d'une gwerz en breton datant des mois ou des années qui suivent la bataille, le texte a une double valeur : outre la célébration d'une victoire bretonne, elle contribue, par sa langue même, à associer Haute et Basse-Bretagne, Bretagne gallèse - francophone donc - et Bretagne bretonnante. En proposant quelques années plus tard " au concours des architectes bretons ",

19. Oléron, Anne et Rondel, Eric, Matignon. 2000 ans d'histoire, Fréhel, Ed. d'Astoure, 1997, p. 186.

20. Il convient cependant d'inscrire cette anglophobie dans la longue durée; LE MOAL, Laurence, « La construction d'un ennemi héréditaire : l'Anglo-Saxon dans les chroniques bretonnes de la fin du Moyen Âge ", Annales de Bretagne et des Pays de l'Ouest, 2001-3, p. 35-56.

21. « Les Bretons et les Anglais sont voisins/mais n'en sont pas moins ennemis/ils ont été mis au monde/pour se combattre à tout jamais "; LA VILLEMARQUE, Théodore Hersart de, Barzaz-Breiz. Chants populaires de la Bretagne, Paris, Delloye, 1867, p. 336. Sur ce point, voir supra, l'article de GullLoREL, Éva, "Chanson politique et histoire... ». 
en l'adressant " à toutes les mairies des principales villes de Bretagne ", en sollicitant l'aide des préfets des cinq départements de la Bretagne historique - et d'eux seuls -, la commission formée à Dinan à l'automne 1857 en vue de l'érection du monument de Saint-Cast s'inscrit dans une logique assez similaire ${ }^{22}$. La chose ne va pas de soi pourtant.

Ainsi, si, pour Frédéric de La Noue, auteur d'un opuscule publié au moment de l'inauguration du monument en septembre 1858, " pour la contrée surtout qui fut le théâtre d'un grand élan national, ce souvenir est un culte " entretenu par " la mère [qui] raconte cette héroïque légende aux fils du matelot qui voyage dans les mers lointaines ${ }^{23}$ ", le folkloriste Paul Sébillot, originaire des environs de Saint-Cast, n'en trouve que bien peu de traces. Celles d'une éventuelle mémoire populaire locale sont des plus ténues. C'est d'ailleurs ce qui le pousse, à l'instar de ses prédécesseurs Luzel, Hersart de La Villemarqué et La Noue, à rédiger lui-même un poème en tenant lieu ${ }^{24}$.

Pourtant, dès les semaines qui avaient suivi la bataille, l'idée d'une victoire bretonne avait pris corps, notamment dans le cadre d'une comparaison - flatteuse - avec les événements cherbourgeois du mois d'août 1758. Un chroniqueur malouin anonyme rapporte ainsi que des soldats britanniques auraient déclaré "que les Normands sont des poltrons et les Bretons des téméraires ${ }^{25}$ ", tandis que, dans les rues de la cité corsaire, l'on chante :

Chez les Normands et les Bretons

Les Anglois ont fait carillon

Voilà la ressemblance.

Les Normands sont de vrais poltrons

De vrais Césars sont les Bretons

Voilà la différence ${ }^{26}$.

Dans un texte trégorrois qui pourrait être contemporain, la victoire est portée au crédit des enfants du pays :

\author{
Allons gwerset Treger, diskoet et c'hoc'h potret \\ Biskoas ' $n$ hon eskopti na so bet poultronet \\ En avant! Potred vad, hag a po war va le \\ Danve-justino ru da rei d'o kroage ${ }^{27}$
}

22. Sur le travail de cette commission, Arch. dép. des Côtes-d'Armor, 1 M 394.

23. La Noue, Frédéric de, Notice..., op. cit., p. 17.

24. SeBillot, Paul, La Bataille de Saint-Cast (1758), Vannes, Lafolye, 1900, 12 p.

25. Lemasson, Auguste, " Nouvelle relation de la descente des Anglais à Saint-Briac et de leur défaite à Saint-Cast en 1758, suivie d'une lettre inédite d'un officier du Fort La Latte ", Mémoires de la Société d'émulation des Côtes-du-Nord, 1940, p. 288.

26. LA BORDERIE, Arthur de, " Chansons inédites relatives aux deux descentes des Anglais en Bretagne en 1758 ", Revue de Bretagne et de Vendée, 1883, p. 92.

27. "Allons, gens du Tréguier, montrez que vous êtes des hommes/Jamais en notre évêché, il n'y eut de poltron/En avant ! vaillants gars, et vous aurez, je vous le jure,/ l'étoffe de justins rouges pour donner à vos épouses "; Chanoine PÉRENNÈs, "Vielles chansons 
Certes, toutes ces paroles n'ont qu'un lointain rapport avec la réalité ${ }^{28}$. L'essentiel n'est pas là, mais plutôt dans le fait que la mémoire de l'événement ait peut-être encore été préservée au moment où le folkloriste Jean-Marie de Penguern l'aurait recueillie dans les années $1840{ }^{29}$. Celle-ci se révèle pourtant très inégalement partagée. Ainsi, en 1858, malgré des sollicitations répétées dans la presse ou par l'intermédiaire des préfets, malgré les efforts de la commission dinannaise présidée par le sous-préfet de la ville pour faire réunir l'argent nécessaire à l'érection d'un monument commémoratif breton ${ }^{30}$, les souscripteurs se concentrent très majoritairement autour de Dinan, de manière plus large dans les Côtes-du-Nord et en Ille-et-Vilaine. Rares sont les habitants du Finistère, de Loire-Inférieure ou du Morbihan à s'engager financièrement : seuls font exception l'évêque de Nantes et quelques aristocrates ${ }^{31}$.

Qu'importe. Il était dit que Saint-Cast serait un " lieu de mémoire " pour tous les Bretons, de Haute comme de Basse-Bretagne.

\section{Saint-Cast, entre Paris et la Bretagne}

Certes, l'image de l'unité de la Bretagne s'élabore très largement face aux Britanniques. Mais la particularité de la construction du " lieu de mémoire " que devient peu à peu Saint-Cast tient au fait qu'il se soit aussi édifié - plus encore peut-être - face au repoussoir représenté par le " Parisien ".

Ce «bleu " presque par nature avait, de fait, aux yeux de certains, déjà sapé les célébrations du centenaire de la bataille. "C'est à Paris que s'est fait le choix du projet " de monument se plaignait ainsi en juillet 1858 Louis de Kerjean dans la Revue de Bretagne et de Vendée, dénonçant l'acceptation du choix fait par l'architecte nantais Bourgerel d'un lévrier

bretonnes ", Bulletin diocésain d'histoire et d'archéologie. Diocèse de Quimper et de Léon, XXXIX, 1939, p. 126 et Collection Penguern, BnF, tome 94, $\mathrm{f}^{\circ}$ 29-32.

28. L'on sait ainsi que les miliciens gardes-côtes trégorrois sont restés en arrière, loin des combats, constituant - avec d'autres - l'essentiel des réserves de d'Aiguillon tout au long de la bataille. Et s'ils ouvrirent le feu, ce fut contre d'autres troupes françaises qu'ils auraient prises pour des Anglais en raison de leurs manteaux de couleur rouge; SAINT Pern-CouËLlan, M. de, "Combat de Saint-Cast ", dans Annuaire dinannais, Dinan, Jean-Baptiste Huart, 1836, p. 217.

29. Comme le note cependant supra GulLLOREL, Éva, "Chanson politique et histoire... ", il convient de rester prudent quant à la datation et à l'origine de certains textes de la collection Penguern. Se reporter aussi, pour d'autres descentes britanniques, à l'étude suggestive de LAURENT, Donatien, "Argadenn ar Saozon. Une descente d'Anglais en Bretagne à la fin du Moyen Âge, d'après un chant de la collection Penguern : Texte authentique ou fabriqué? ", Regards étonnés. Mélanges offerts au professeur Gaël Milin, Brest, CRBC, 2003, p. 289-298.

30. Le brouillon de l'appel à souscription, en date du 15 décembre 1857, évoque une " idée toute bretonne [qui] doit avoir du retentissement dans toute la Bretagne, car ce n'est pas un de ces faits dont la gloire appartiendra à une partie restreinte du pays que ce monument rappellera, mais bien un souvenir qui doit être précieux pour tous les cours bretons "; Arch. dép. des Côtes-d'Armor, 1 M 394.

31. Voir les listes publiées en 1858 dans le journal local, Union malouine et dinannaise. 
pour représenter la Bretagne : " une monstruosité " écrivait-il, " contre [laquelle] la Bretagne entière ne peut manquer de protester ${ }^{32}$ „. La chronique suscita dans les jours qui suivirent une réponse violente de J. Bazouge, rédacteur de l'Union malouine et dinannaise, le journal local, qui saluait lui l'option choisie par la commission dinannaise en charge de l'édification du monument de Saint-Cast de s'en remettre au - certes parisien... - Conseil général des bâtiments civils. Le journaliste en profitait cependant pour dénoncer, de manière plus profonde, les germes de division portés par l'idéologie défendue par Louis de Kerjean, jusqu'au nom de sa revue, associant la Bretagne à une Vendée dont « les tristes souvenirs [...] sont de ceux qu'il ne faut rappeler que pour inspirer l'horreur de la guerre civile, que pour gémir sur les deuils de la patrie ${ }^{33}$ ». Historien réputé, Arthur de La Borderie, directeur de la revue, ne pouvait manquer de prendre part au débat. Dans une chronique publiée une première fois dans le très conservateur Journal de Rennes le 16 août 1858, il se lançait dans une longue protestation contre " ce prétendu symbole national " proposé aux Bretons en ce lévrier rappelant " un acte de félonie exécuté au profit d'un prince vendu aux Anglais ": celui du lévrier de Charles de Blois à Auray en $1364^{34}$. Surtout, cette tribune était pour lui l'occasion de rappeler qu'en 1758, le duché de Bretagne " était uni à la Monarchie, il n'était pas absorbé ${ }^{35}$ ".

De nature très indirectement esthétique, ce débat, on le voit, en suggérait d'autres, bien plus généraux, notamment sur la nature des rapports entre la France et la Bretagne, entre Paris et l'ancienne province. En cela, la bataille de Saint-Cast s'inscrivait parfaitement dans les perspectives des leaders légitimistes bretons : une France décentralisée, celle des provinces, dominée par ses plus grandes familles. Cela passait, bien entendu, par le soutien apporté à l'édification d'un monument à Saint-Cast - la Revue de Bretagne et de Vendée n'avait-elle pas accordé une souscription de 50 F? -, un monument cependant différent de celui choisi à Paris. Cela passait aussi par la réaffirmation du rôle tenu par la noblesse bretonne : nous y reviendrons. Cela passait enfin et surtout par le rappel de la " celtitude " bretonne, source d'une plus grande proximité avec certains des cousins britanniques qu'avec Paris, suggérée notamment par l'une des légendes les plus solidement ancrées concernant Saint-Cast.

32. KeRJean, Louis de, "Chronique ", Revue de Bretagne et de Vendée, 1858, p. 86-87.

33. Bazouge, J., "Causeries dinannaises ", Union malouine et dinannaise, 25 juillet 1858.

34. LA BORDERIE, Arthur de, " Le Chien de Saint-Cast et ses défenseurs ", Revue de Bretagne et de Vendée, 1858, p. 270. Sans doute n'est-il guère utile de revenir ici sur le fond de ce débat. Notons simplement que des lévriers ont, de manière récurrente, pu être utilisés pour représenter la Bretagne avant 1858.

35. Sur les conceptions politiques de La Borderie, plus complexes qu'on ne le dit souvent, DENIS, Michel, " Arthur de La Borderie, inspirateur du nationalisme breton ", Bulletin et Mémoires de la Société archéologique et historique d'Ille-et-Vilaine, 2002, p. 190-207. 
À son origine, l'on trouve Saint-Pern Couëllan à qui des témoins interrogés dans les années 1820 auraient dit que des miliciens bas-bretons, en marche vers le champ de bataille, auraient été surpris de tomber sur des soldats britanniques chantant l'une de leurs propres chansons. Ces soldats - des Gallois - auraient été tout aussi ahuris d'entendre leur hymne national dans la bouche de l'ennemi. Malgré les ordres donnés, de part et d'autre, de faire feu, les deux groupes de soldats seraient tombés dans les bras les uns des autres, "les descendants des vieux celtes [renouvelant] sur le champ de bataille, les liens de fraternité qui unissaient jadis leurs pères ${ }^{36}$ ". À peine ce récit était-il mis en circulation que La Villemarqué redécouvrait - semble-t-il - à son tour une chanson concernant Saint-Cast rappelant la même étrange rencontre ${ }^{37}$. Malgré la place qu'elle prit rapidement dans la " querelle du Barzaz-Breiz " qui divisa le mouvement régionaliste à partir des années $1860^{38}$, cette légende n'en passe pas moins dans le " folklore " des deux côtés de la Manche, permettant aux Gallois comme aux Bretons de démontrer qu'ils ne constituent pas seulement des régions mais aussi de véritables nations, avec leurs propres langues, leurs histoires partagées, des nations comme tant d'autres oppressées par des " étrangers " - Anglais ou Français -, sans même parler de l'appartenance à une même " famille " de nations celtes ${ }^{39}$.

De cette appropriation bretonne de l'événement devait découler la construction du mythe d'une victoire bretonne, en quelque sorte " indigène ". Elle avait cependant besoin d'un héros et, plus encore, d'un héros autochtone. Il convenait donc pour cela, dans un premier temps, de disqualifier celui, allochtone, qu'avait pu, un temps, incarner le duc d'Aiguillon.

\section{Le duc d'Aiguillon, un anti-modèle allochtone}

En 1758, les lauriers furent si largement distribués aux vainqueurs qu'ils revinrent même aux miliciens bas-bretons pourtant bien peu actifs lors de la bataille elle-même. Très rapidement cependant, certains en vinrent à remettre en cause le rôle du duc d'Aiguillon et, par ricochet, la contribution de la Couronne de France à la victoire. Cette disqualification du commandant en chef repose sur trois éléments principaux.

\section{D'Aiguillon, La Chalotais et la belle meunière}

L'Affaire de Bretagne, dans laquelle le commandant en chef, comme représentant du roi, s'opposa aux états et au parlement de Rennes, insti-

36. Saint Pern-CouËllan, M. de, "Combat... », art. cit., p. 235.

37. Voir sur ce point, supra, l'article de GulLLorel, Eva, "Chanson politique et histoire...".

38. Lотн, Joseph, "Une chanson inédite sur le combat de Saint-Cast ", Annales de Bretagne, XII, 1896-1897, p. 607-617 et GouRVIL, Francis, "Bretons et Gallois à la bataille de Saint-Cast (1758) ", Nouvelle Revue de Bretagne, 4, 1947, p. 265-275.

39. AR C'HALAN, Reun, "History or Legend? An Episode of the Battle of Saint-Cast ", Bro Nevez, n 35 , August 1990, p. 29. 
tutions symbolisant tout autant le particularisme breton que les privilèges de la noblesse, marque indéniablement un tournant dans l'appréciation des mérites de chacun lors des combats de 1758.

Passons ici sur les détails de cette affaire ${ }^{40}$, pour ne retenir que l'une des questions - certes mineure - qu'elle pose, celle des rivalités personnelles opposant d'Aiguillon à La Chalotais, incarnation de l'opposition de la noblesse bretonne au "despotisme " monarchique. Très tôt en effet, à en croire le baron de Bésenval, témoin des événements, circula une anecdote selon laquelle, alors que quelqu'un vantait la bravoure du commandant en chef à La Chalotais, le procureur-général aurait répondu : " Notre commandant a vu l'action, d'un moulin, où il s'est couvert de farine, en guise de lauriers ${ }^{41}$. " Connu pour être un " homme à femmes ", d'Aiguillon, qui avait installé son poste de commandement sur une position dominant la plage de Saint-Cast, près du Moulin d'Anne, se serait, selon la rumeur, réfugié dans l'édifice pour se protéger du bombardement naval des Britanniques. Y découvrant la fameuse Anne, il aurait tenté de la violenter. Sa distraction aurait par ailleurs permis au gros des forces britanniques de s'échapper, seule l'action du commandant de l'aile gauche, le comte d'Aubigny, ayant évité à l'arrière-garde de regagner, elle aussi, les navires. Même s'il est peu probable que La Chalotais ait jamais lui-même prononcé ces mots, l'histoire semble déjà connue pendant l'Affaire de Bretagne. Elle apparaît dans un libelle manuscrit anonyme de 1766, l'un de ces innombrables pamphlets qui firent de cette querelle politique une affaire véritablement populaire ${ }^{42}$. On le sait désormais : l'histoire n'est guère plausible, notamment parce qu'il n'y avait pas d'Anne dans le moulin éponyme ${ }^{43}$.

40. Pour de plus amples précisions, se reporter à QUÉNIART, Jean, La Bretagne au XVII siècle (1675-1789), Rennes, Ouest-France, 2004, p. 86-110 ou ROTHNEY, John (dir.), The Brittany Affair and the Crisis of the Ancien Régime, Oxford, Oxford UP, 1969.

41. BéSENVAL, baron de, Mémoires de M. le baron de Bésenval... écrits par lui-même, imprimés sur son manuscrit original et publiés par son exécuteur testamentaire, Paris, 1805 vol. 2, p. 172. Selon PoCQUET, Barthélemy, Histoire de Bretagne, op. cit., p. 274, qui le juge " absolument calomnieux ", La Chalotais aurait déclaré qu'à Saint-Cast, " nos soldats se sont couverts de gloire, et le petit duc... de farine"

42. Nouveau recueil de documents inédits sur la campagne et la bataille de Saint-Cast (septembre 1758), Saint-Brieuc, Société archéologique et historique des Côtes-du-Nord, 1885, p. 275-283. Le fait que ce recueil soit dû à Arthur de La Borderie n'est sans doute pas anodin ici, ainsi qu'en témoigne la virulence avec laquelle il réagit après la publication, en juin 1898 , dans la Revue de Bretagne et de Vendée qu'il dirige pourtant, d'un " compte-rendu absolument laudatif, sans aucune réserve " de l'ouvrage que Marcel Marion a consacré au duc d'Aiguillon (La Bretagne et le duc d'Aiguillon, Paris, Fontemoing, 1898). "Sur ce livre, mon opinion est diamétralement l'inverse de celle du compte-rendu " écrit-il dans le $\mathrm{n}^{\circ}$ d'octobre; et de préciser en janvier 1899 que " le livre de M. Marion est, d'un bout à l'autre, un réquisitoire amer, passionné, violent, et selon moi, parfaitement injuste, à l'adresse de tous les Bretons qui soutinrent contre les attaques de l'arbitraire, les libertés de la province " et « une apologie constante, ardente et non moins injuste du despotisme ministériel de ce triste gouvernement de Louis XV " (LA BORDERIE, Arthur de, "Contre les panégyristes du duc d'Aiguillon ", Revue de Bretagne et de Vendée, oct. 1898, p. 241-246 et janv. 1899, p. 5-12.

43. En 1758, le meunier est un certain Louis Renault, célibataire; LA MoTTE-RougE, Daniel de, Saint-Cast, son site, son histoire, Saint-Brieuc, Les Presses bretonnes, 1950, p. 17 et, du même, Saint-Cast. Son histoire, Saint-Cast, Syndicat d'Initiative, 1964, p. 10. 
Le fait n'en est pas moins régulièrement repris, perdurant, aujourd'hui encore, jusque dans le nom d'un des salons de thé de la rue principale de Saint-Cast, La belle meunière.

\section{Hésitations de d'Aiguillon, action décisive des volontaires bretons}

La critique aristocratique de la conduite du commandant en chef à SaintCast va cependant bien au-delà de ses frasques extraconjugales, gagnant un terrain plus strictement militaire.

L'on doit ainsi à Maurice de Couëssin, l'un des volontaires bretons servant en avant de la colonne de d'Aubigny, un mémoire accordant à ces mêmes volontaires l'action décisive qui permit de décider du sort de la bataille du 11 septembre. Bloqués par le feu de l'artillerie navale britannique en débouchant sur la plage depuis un cheminement couvert, les soldats de l'aile gauche auraient trouvé refuge derrière les dunes, rien ne pouvant les en faire sortir. D'Aubigny se serait alors tourné vers les nobles bretons, leur demandant de donner l'exemple. Chargeant à travers les $300 \mathrm{~m}$ de plage les séparant des lignes britanniques, les volontaires auraient finalement été suivis par les grenadiers. Ce serait cette action, selon Couëssin, qui aurait décidé de l'issue de la bataille, la Navy étant contrainte de cesser ses bombardements une fois soldats français et britanniques au contact. D’Aubigny et les volontaires bretons seraient donc, bien plus que d'Aiguillon, les véritables vainqueurs de Saint-Cast.

Les reproches de Couëssin au commandant en chef ne s'arrêtent pas à son inaction supposée, la critique touchant à la capacité même du duc à "saisir " la bataille afin d'influer sur son déroulement. Ainsi d'Aiguillon n'aurait-il pas suffisamment fait surveiller le camp des troupes de Bligh lors de la nuit du 10 au 11 septembre 1758; il n'aurait fait que trop tardivement sa jonction avec d'Aubigny au matin du 11; il n'aurait pas fait donner l'artillerie à temps, empêchant une victoire plus large encore ${ }^{44}$. Quel crédit accorder à ces critiques, formulées bien après les événements? Rappelons, simplement, que Couëssin, parce que noble, était membre de droit des états de Bretagne, de ces états qui s'opposèrent des années durant à la politique de d'Aiguillon. En cela, la diffusion de ces accusations peut être comprise comme un élément supplémentaire de l'Affaire de Bretagne. Pourtant, bien que connu de certains milieux en Bretagne dès les années précédant la Révolution, le mémoire ne fut publié qu'en 1836, à l'initiative du député légitimiste de Dinan, Saint-Pern Couëllan. Lui-même lié à plusieurs acteurs de la bataille, sa famille avait, elle aussi, œuvré contre le "despotisme royal " incarné par d'Aiguillon dans le cadre des états. Aussi son introduction à la publication du texte de Couëssin ne se contente-t-elle pas de minimiser le rôle de d'Aiguillon.

44. Saint Pern-CouËllan, M. de, "Combat... ", art. cit., p. 221-225. 
Le notable dinannais s'en prend en effet plus largement à l'action des troupes régulières : "C'étaient les Bretons eux-mêmes, qui, aidés de quelques régiments, défendaient leurs foyers ; c'étaient les milices, nos concitoyens de toutes les classes, qui, surpris au milieu de leurs familles par le cri de guerre, étaient accourus pour repousser l'ennemi " écrit-il notamment ${ }^{45}$. En plein XIX ${ }^{\mathrm{e}}$ siècle, Saint-Pern Couëllan rejoue en quelque sorte l'Affaire de Bretagne, notamment parce qu'elle reste pleine de sens à ses yeux, matérialisant les liens de continuité unissant le " despotisme royal " incarné par d'Aiguillon et la centralisation de l'État post-révolutionnaire - plus encore en ces années 1830, alors que Louis-Philippe, roi libéral se réclamant des idéaux de 1789 , fils d'un régicide, règne sur le pays. Tandis que le premier avait tenté de détruire la Bretagne en tant qu'entité politique, le second avait réussi à détruire ces corps intermédiaires, chers aux légitimistes, qu'avaient été les états et le parlement de Bretagne, déniant à la noblesse toute place spécifique dans le gouvernement de la province. Les affaires bretonnes, insinuait Saint-Pern, étaient mieux gérées lorsqu'elles l'étaient par les leaders naturels de la province, qu'incarnaient, entre autres, ses propres aïeux. Son récit de Saint-Cast se devait donc d'appuyer cette vision de l'histoire provinciale.

Or, durant une bonne partie du XIX ${ }^{\mathrm{e}}$ siècle, c'est cette version de la bataille, selon laquelle les paysans se seraient soulevés à l'appel de leurs leaders naturels, qui fit figure de vulgate en Bretagne.

\section{Noblesse et paysannerie de Bretagne unies, de 1758 à 1793}

Le texte de Couëssin, repris, après Saint-Pern, par les continuateurs d'Ogée en $1853^{46}$, fournit les bases des récits qui suivirent. Aussi, à l'époque du centenaire de la bataille, la présence des troupes royales était comme oubliée, jusque dans la rhétorique des prises de position entourant les célébrations.

L'Union malouine et dinannaise, le journal local, se fit l'écho des propos de Saint-Pern dans ses articles concernant l'inauguration de la colonne du centenaire : " au bruit terrible des canons de l'étranger " lit-on dans l'édition 12 septembre 1858, " les gentilshommes, la milice, les volontaires de Bretagne, appuyés de quelques régiments français, accouraient, nombreux et vaillants, sur les rivages de Saint-Cast : le château, la chaumière fournirent généreusement leurs contingents de braves ${ }^{47}$ ". " Appuyés de quelques régiments français " : l'expression laisse songeur, seule une soixantaine de volontaires bretons - nobles pour la plupart - ayant pris part au combat en

45. Ibidem, p. 187-188.

46. OgÉE, Jean-Baptiste, Dictionnaire historique et géographique de Bretagne, Rennes,

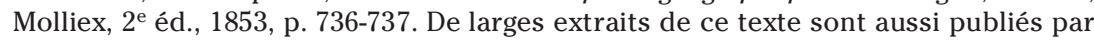
l'hebdomadaire Le Publicateur des Côtes-du-Nord du 16 septembre 1837, à l'occasion du $79^{\mathrm{e}}$ anniversaire de la bataille.

47. L'Union malouine et dinannaise, dimanche 12 septembre 1858. 
une mobilisation qui n'est pas sans rappeler celle de l'arrière-ban en $1746^{48}$. D'autres vont plus loin pourtant, tel Sigismond Ropartz, éditeur en 1858 d'une impressionnante série de documents relatifs aux événements :

" Des extrémités de la province, par des chemins impraticables, à travers les landes, à travers les champs dont les clôtures sont abattues, soldats, gardes-côtes, volontaires, gentilshommes, bourgeois et paysans, forçant les étapes, marchent la nuit comme le jour; toute une petite armée s'est assemblée $^{49}$."

Plus de régiments ici, non plus que chez Frédéric de La Noue, dont la famille avait pourtant pris le parti de d'Aiguillon aux états, et dont les commentaires sur le commandant en chef étaient donc plus nuancés : cela ne l'empêche pas d'écrire que « la Bretagne, en glorifiant le souvenir de SaintCast, célèbre un souvenir qui lui appartient sans conteste ${ }^{50}$ ".

Une cinquantaine d'années plus tard, pour l'historien Aurélien de Courson, non seulement la noblesse bretonne a gagné la bataille « presque seule ", mais les Bretons ont pris à leur compte le fardeau tout entier de la défense nationale: "Il est curieux de voir une province, toujours si brutalement, si injustement traitée par les prédécesseurs de Louis XV, se lever soudain, avec un superbe élan de patriotisme pour chasser l'ennemi du territoire, alors que, dans tout le reste de la France, l'honneur, le désintéressement et l'esprit militaire semblent avoir complètement disparu " notet-il par exemple ${ }^{51}$. Le type d'histoire rédigé par Courson peut ainsi se lire comme une apologie d'un - mythique - Ancien Régime breton, et en cela comme une condamnation de l'État français, qu'il soit absolutiste ou postérieur à $1789^{52}$. Selon lui, non seulement les gouvernements successifs de la France ont trahi la Bretagne, mais ils ont trahi la France elle-même. En cela, les Bretons seraient les véritables patriotes français, notamment par leur loyalisme envers une cause que la France aurait, elle, oubliée. Se confondraient ainsi, en un même objectif, l'action des nobles bretons volontaires en 1758 et celle de leurs descendants ayant pris part aux Chouanneries, tels les ancêtres de Courson lui-même. La Contre-Révolution fut d'ailleurs particulièrement active sur les côtes nord de la Bretagne, impliquant, d'une manière ou d'une autre, bon nombre des familles nobles de la région. $\mathrm{Si}$ Couëssin lui-même émigra à Jersey, des membres de sa famille combattirent aux côtés du chef chouan Boishardy, dont le père servit d'ailleurs comme volontaire à Saint-Cast. Le grand-père de Saint-Pern Couëllan, frère du commandant de la réserve lors de la bataille de 1758, n'échappa à la

\footnotetext{
48. Sur ce point, PERRÉON, Stéphane, L'Armée en Bretagne au XVIII siècle. Institution militaire et société civile au temps de l'intendance et des États, Rennes, PUR, 2005, p. 131-136.

49. Ropartz, Sigismond, "Préface ", op. cit., p. vI.

50. La Noue, Frédéric de, Notice..., op. cit., p. 97.

51. Courson, Aurélien de, Descente des Anglais à Saint-Cast en 1758, Vannes, Lafolye, 1903. p. VI.

52. Sur les liens entre histoire et régionalisme, GuIOMAR, Jean-Yves, Le bretonisme..., op. cit.
} 
guillotine qu'en raison de sa mort à l'hôpital; sa femme, elle, fut exécutée ${ }^{53}$. Les nobles bretons qui, en dépit de déceptions successives, auraient fourni les principaux défenseurs du royaume en 1758, se devaient d'être aussi à la tête des défenseurs de la cause royale en 1793. La France, qui les avait trahis dans l'Affaire de Bretagne, continuait d'ailleurs à le faire; en 1793 ou 1795 comme en 1830 ou 1870/1871, au moment de l'" affaire » de Conlie.

Filtrée par la mémoire de la Révolution, l'histoire de la bataille faisait désormais aux yeux de certains, des miliciens de 1758 des sortes de "préchouans ". La chose est particulièrement nette sur les quelques représentations iconographiques des $\mathrm{XIX}^{\mathrm{e}}$ et $\mathrm{XX}^{\mathrm{e}}$ siècles. Ainsi, sur le vitrail commémoratif de l'église paroissiale de Saint-Cast, des hommes en costume traditionnel bas-breton, armés de faux, entourent des officiers royaux. La vision ainsi donnée de la Bretagne et de son unité est des plus intéressantes. L'unité entre Haute et Basse-Bretagne est en effet une fois encore implicitement rappelée; mais cette unité est aussi celle liant toutes les composantes d'une société conçue de manière holiste : point de tensions sociales ici, de « lutte des classes ", mais un tout indifférencié associant " nos concitoyens de toutes les classes " selon Saint-Pern, " gentilshommes, bourgeois et paysans " selon Ropartz ${ }^{54}$, luttant de concert pour la Bretagne mais aussi - le vitrail le rappelle indirectement - pour la " vraie " foi.

Des paysans patriotes, répondant à l'appel du tocsin pour combattre l'envahisseur hérétique, sous le commandement des élites locales traditionnelles : la présentation des faits relatifs à la défense du gué du Guildo les 8 et 9 septembre 1758 apparaît, plus encore que la bataille du 11 septembre, emblématique de cette conception de la société.

\section{Forger un héros autochtone : Rioust, un Léonidas breton}

Se focaliser sur la défense du gué du Guildo, deux jours avant la bataille, permet à une partie des historiens bretons de minorer encore un peu plus la contribution des troupes royales - à leurs yeux " françaises ", non-bretonnes donc - à la victoire. L'affrontement de milliers d'hommes sur la plage de Saint-Cast fait alors figure de simple appendice à l'action menée par quelques dizaines de gardes-côtes sur les rives de l'Arguenon : c'est là, en ces véritables Thermopyles bretonnes, que l'issue de la bataille se serait

53. Sur l'histoire des familles des volontaires bretons, La CondaminE, Pierre de, Un jour d'été à Saint-Cast, Guérande, Le Bateau qui vire, 1977, p. 127-295.

54. SAINT PERn-Coü̈lLAN, M. de, "Combat... ", art. cit., p. 187-188 et RopartZ, Sigismond, "Préface", op. cit., p. vI. Cette vision " bretonne " de la bataille de Saint-Cast est aussi largement véhiculée dans les travaux d'histoire grand public, même lorsque ceux-ci n'ont pas été écrits dans une perspective régionaliste. Destiné aux touristes, le guide de Danycan de L'Espine suggère ainsi à ses lecteurs un détour par Saint-Cast, « lieu historique puisqu'il a donné le nom à cette bataille si glorieuse pour la province bretonne, car ce furent ses enfants, nobles et paysans, qui, à la voix de leurs chefs, vainquirent les troupes de l'armée régulière de la Grande-Bretagne "; DANYCAN DE L'EsPINE, Eugène, Pléneuf et ses environs : guide du baigneur, Paris, L'œuvre de Saint-Paul, 1883, p. 93. 
décidée. Conduits par des nobles et notables locaux, les paysans auraient contenu l'ensemble de l'armée britannique, permettant à d'Aiguillon de rassembler ses troupes afin de porter le coup mortel. On le voit, cette action a tout pour s'accorder aux schémas idéologiques des historiens bretons monarchistes, bien plus que la bataille elle-même.

Encore fallait-il trouver un Léonidas pour ces Thermopyles armoricaines. La question préoccupa les auteurs bretons pendant près de deux siècles.

\section{Un ou des Léonidas?}

Créer un tel héros prit du temps. Aux lendemains immédiats des événements, les honneurs de cette action reviennent à trois petits nobles des environs, de la Motte-Ville-ès-Comte, Langlais de Prémorvan et La Planche de Kersula ${ }^{55}$.

Quand, en 1759, les États de Bretagne dressent la liste de ceux dont les services méritent une récompense, les trois hommes y figurent comme " défenseurs du Guildo ». Certes, ils sont originaires de la rive droite de l'Arguenon, alors que la défense s'organisa sur la rive opposée. Certes, selon des témoins, les trois nobles n'ont participé que tardivement aux opérations des 8 et 9 septembre. Mais leurs noms apparaissent dans plusieurs des plus anciens récits de la bataille, quand bien même l'essentiel de cette action semble avoir reposé sur les épaules de personnages de moindre importance, tels Galiot, cavalier de maréchaussée de Lamballe, Gabriel Lemasson, charpentier de marine au Guildo, ou encore Jean-François Rébillard, petit officier de Matignon à qui revenaient cependant les fonctions de « capitaine de paroisse " au sein de la milice garde-côtes locale.

La confusion des récits contemporains révèle d'ailleurs sans doute, pour une part, une certaine réalité. Plusieurs groupes différents, plus ou moins informels, firent probablement le coup de feu ici : fort logiquement les gardes-côtes locaux, mentionnés par l'aubergiste du lieu et la maréchaussée de Lamballe, mais aussi une troupe de chasseurs conduite justement par La Motte Ville-ès-Comte. Quoi qu'il en soit, personne n'eut sans doute les moyens de superviser et coordonner tous ces groupes. Aussi le crédit accordé aux uns et aux autres pour leur participation à cette action dépendit-il très largement des témoins interrogés sur ce point, du village d'où ils venaient, du moment auquel ils étaient arrivés à proximité du gué.

Vingt ans après la bataille pourtant, un nouveau personnage apparut en la personne de Jacques Pierre Rioust des Villes-Audrains, selon ses propres

55. Le chevalier Joseph Amaury de La Motte, né en 1721, était seigneur de la Ville-èsComte en Trégon et Joseph Marie de Langlais seigneur de Prémorvan en Saint-Potan et du Plessis-Méen en Hénanbihen. Jean-François de La Planche de Kersula s'était déjà mobilisé contre les Anglais pour la défense de Saint-Malo en juin 1758; son action au Guildo lui valut une rente viagère de $200 \mathrm{~L}$. de la part des états de Bretagne; sur ces trois hommes, La Condamine, Pierre de, Un jour d'été...., op. cit., p. 249-253. 
mots « bourgeois " et propriétaire à Matignon. En 1778 en effet, il écrivait au secrétaire d'État à la Guerre pour se plaindre de n'avoir jamais reçu la récompense à lui promise par d'Aiguillon pour avoir conduit la défense du Guildo : une lieutenance de cavalerie. Selon ses termes, les calomnies colportées par certains, dont Rébillard, suggérant qu'il n'aurait pas pris part aux événements expliqueraient sa démarche. Ce courrier initia en fait une véritable campagne que Rioust orchestra de main de maître, mobilisant tous ses contacts, allant jusqu'à leur fournir des modèles de missives à recopier et envoyer à l'intendance de Bretagne, dans le but de se voir accorder des lettres de noblesse. Des Villes-Audrains y mettait notamment en avant qu'il était celui qui avait déclenché l'alarme dans la nuit du 7 au 8 septembre 1758, qui avait ensuite conduit les hommes de Matignon et de Saint-Cast jusqu'au Guildo. Tout en admettant que toutes les personnes mentionnées habituellement dans les récits étaient bien là, il précisait cependant qu'elle n'étaient arrivées que tardivement et s'étaient contentées de suivre ses ordres. Quant à Rébillard, qui revendiquait pour lui-même - de manière ridicule selon Rioust - les fonctions de commandant, accusant d'ailleurs ce dernier d'avoir détourné à son profit les fonds accordés par les états de Bretagne aux habitants de Matignon dont les biens avaient été brûlés ou volés par les Britanniques, il était "le plus pauvre homme de Matignon ": comment croire, dans ces conditions, que des nobles des environs aient pu prendre leurs ordres d'un tel personnage ${ }^{56}$ ?

Cette campagne parvint à convaincre l'intendant qui soutint sa réclamation. La famille Rioust dut cependant attendre jusqu'en 1816 pour que Frédéric Auguste, le fils de Jacques, soit enfin anobli ${ }^{57}$. Tout était prêt alors, pour asseoir cette position de héros.

\section{Rioust, héros consensuel}

Ce n'est qu'au lendemain des guerres de la Révolution et de l'Empire que la publicité du rôle de Rioust dans les combats du Guildo prit une certaine importance, notamment grâce à la diffusion par sa famille de son " journal ", un document écrit longtemps après les événements cependant. À compter des années 1830, ce texte est en effet connu d'un plus large public, au gré de ses publications successives dans l'Annuaire dinannais (1838), l'Annuaire des Côtes-du-Nord (1855) ou le recueil de documents dû à la Société archéologique des Côtes-du-Nord (1858). L'ampleur de cette

56. Sur la légende de Rioust, MAcé, Antonin, Le Passage du Guildo ou un Léonidas Breton. Episode de la Guerre de Sept Ans, Paris, Imprimerie impériale, 1866. Pour sa déconstruction, se reporter à CHENu, J., "Le passage des Anglais au Guildo en 1758 ", Mémoires de la Société d'émulation des Côtes-du-Nord, 1979, p. 62-89. Les documents contemporains révèlent que, si plusieurs personnes se considèrent comme les organisateurs de la défense du gué, seuls Ville-ès-Comte et Rébillard se voient reconnaître ce rôle sans réelle contestation.

57. Sur la famille Rioust, FroTIER DE LA MESSELIÈRE, Henri, Filiations bretonnes (1650-1912). Tome IV, Mayenne, J. Floch, 3e éd., 1976, p. 602-604. 
" campagne de presse " contribua à noyer les autres héros du Guildo sous ses flots, expliquant pour une part que la plupart des historiens des XIX et $\mathrm{xx}$ e siècles aient accordé à Rioust le rôle prépondérant qu'il s'était lui-même donné dans les combats, sans pour autant d'ailleurs empêcher ces mêmes historiens de reconnaître qu'aucun témoin contemporain ne lui ait consenti avoir tenu ce rôle ${ }^{58}$.

Le fait que " bleus " et « blancs " puissent se retrouver dans sa célébration explique sans doute la postérité de Rioust en tant que figure emblématique de la "résistance " bretonne. Le " bourgeois de Matignon " se révélait en effet un héros bien plus consensuel que d'autres, concentrant des qualités pouvant satisfaire l'un et l'autre camp. Si son nom de famille fut au cours du XIX ${ }^{\mathrm{e}}$ siècle associé à un Parti de l'Ordre auquel ses descendants fournirent nombre d'élus ${ }^{59}$, ses enfants avaient l'avantage de ne pas s'être engagés dans la Contre-Révolution, au contraire de ceux de ses rivaux, Ville-ès-Comte et La Planche, morts sur l'échafaud en 1793 et 1795 ou ayant émigré. Bon catholique - il écrivit avoir, sur le chemin du Guildo, suivi la messe en ce jour de fête de la Nativité de la Vierge -, il pouvait cependant contenter les anticléricaux : n'avait-il pas fait ouvrir le feu sur un carmélite du Guildo qui se proposait de parlementer au nom des Britanniques? Parce qu'il n'était pas noble, il n'en était sans doute que plus facilement acceptable par les républicains, même s'il épousa une jeune aristocrate. Enfin, Rioust n'avait rien du serviteur de l'État français : ni fonctionnaire, ni militaire, il n'était qu'un simple " autochtone " ayant pris les armes pour défendre sa propriété et sa communauté ${ }^{60}$.

La capacité du " bourgeois de Matignon " à transcender les divisions politiques bretonnes est illustrée par l'appui enthousiaste qu'il reçut de manière posthume de la part des frères Sébillot comme de celle de Pocquet du Haut-Jussé, continuateur de La Borderie, pourtant en désaccord politique. Figure locale du républicanisme, Pierre Sébillot, maire de Matignon, n'avait pas hésité à faire expulser les ordres religieux de sa commune en 1882 ou à instituer la célébration du 14 Juillet, deux décisions éminemment symboliques. Mais c'est aussi et surtout pendant son mandat qu'Auguste Lemoine, artiste local et ami de Paul Sébillot, fit don à la mairie d'un tableau représentant la rencontre de Rioust et de d'Aiguillon après la

58. Sur ce point, se reporter, par exemple, à LEMASSON, Auguste, La Descente des Anglais..., op. cit., p. 34 .

59. Frédéric-Marie-Ange Rioust de Largentaye (1797-1856), son petit-fils, fut conseiller général des Côtes-du-Nord et représentant du Peuple de 1849 à 1852. Le fils de celui-ci, Marie-Ange-Julien Charles, fut lui aussi conseiller général des Côtes-du-Nord et représentant puis député de 1871 à sa mort. Frédéric, l'un de ses fils, fut député de 1884 à 1910, son frère Jacques se contentant des fonctions de conseiller général.

60. Notons que l'émergence de Rioust comme figure héroïque est concomitante de la construction d'un autre "mythe ", celui de la traîtrise d'un autre homme du peuple, Grumelon; sur cet aspect, CHENU, J., " Le passage des Anglais au Guildo en 1758 ", Mémoires de la Société d'émulation des Côtes-du-Nord, 108, 1980, p. 62-89 et HopKIN, David, LaGADEC, Yann et Perreon, Stéphane, "The Experience and Culture of War... ", art. cit. 
bataille ${ }^{61}$. Le peintre semble s'être inspiré de la toile - bien plus célèbre - de Daniel Maclise figurant la rencontre de Wellington et de Blücher à la Belle Alliance, le 18 juin 1815. Les deux hommes y apparaissent à cheval, sur le même plan, comme si le "bourgeois " de Matignon et le commandant en chef de la province étaient socialement et militairement égaux. Mais d'Aiguillon occupe ici la position de Blücher, l'homme qui arriva trop tard pour emporter la décision à Waterloo... En 1911, Lemoine, soutenu par Paul Sébillot, lançait même une souscription pour une statue devant orner la place principale de Matignon, une statue associée à un monument commémoratif à élever dans le même temps au Guildo et à replacer dans le contexte " statuomaniaque " de la III ${ }^{e}$ République ${ }^{62}$. Une maquette fut même conçue, avant que la guerre vienne interrompre ces efforts de " matérialisation " de la mémoire ${ }^{63}$. Le fait que les assertions de Rioust n'aient pas été prouvées était pourtant connu des Sébillot et de Lemoine. Paul Sébillot avait même activement questionné la mémoire locale sur le rôle du bourgeois sur les rives de l'Arguenon, sans jamais réussir à établir le lien entre son nom et les événements de $1758^{64}$.

La chose ne semble pas avoir outre mesure choqué la foi des Sébillot, pas plus d'ailleurs que celle de Barthélemy Pocquet, le très conservateur, catholique, anti-républicain et anti-dreyfusard directeur du Journal de Rennes ${ }^{65}$. Dans l'imposante Histoire de Bretagne qu'il rédige avec La Borderie dans les années 1890-1910, le récit de la défense du Guildo occupe de manière très révélatrice 4 pages, contre 3 seulement pour la bataille de Saint-Cast proprement dite ${ }^{66}$. Pocquet, à l'instar de son illustre collaborateur, était pourtant un historien de premier plan ${ }^{67}$ : sans doute savait-il que les histoires rédigées en Grande-Bretagne ne faisaient pour la plupart

61. Aujourd'hui, ce tableau est conservé à la mairie de Matignon.

62. AgUlHON, Maurice, "La statuomanie et l'histoire ", Ethnologie française, 1978, p. 145172 rappelle le " grand déferlement " que constituent les années 1870-1914 pour la statuaire républicaine, associant aux grands hommes de la République, les héros d'un passé plus ou moins lointain dans un contexte marqué par le nationalisme.

63. TRÉGUY, Émile Le Guildo, Saint-Brieuc, Fr. Guyon, 1913, p. 178.

64. SÉBILlot, Paul, Légendes locales de Haute-Bretagne, Nantes, Société des bibliophiles bretons et de l'histoire de Bretagne, 1900, vol. 2, p. 144. En fait, Sébillot trouve trace d'une mémoire locale de l'action de Rioust, lors de la bataille du 11 septembre cependant, et non à l'occasion de la défense du Guildo.

65. Rappelons que cet avocat, docteur en droit, fut des volontaires de l'Ouest en 18701871 avant de devenir chef de cabinet du préfet de Haute-Marne après le 16 mai 1877. Démis après la crise de cette année, il enseigne à l'Université catholique de Lyon avant de diriger le Journal de Rennes à compter de 1878, suite à la mort de son père. Ce catholique engagé est alors chevalier de l'ordre de Saint-Grégoire-le-Grand et titulaire de la médaille des Combattants de 1870-1871.

66. PocQuet, Barthélemy, Histoire de Bretagne, Rennes, Plihon et Hommay, 1914, T. VI, p. $247-261$.

67. Il n'est guère besoin de rappeler ici ses nombreuses publications; signalons, entre autres, l'ouvrage qu'il signe sur Le pouvoir absolu et l'esprit provincial. Le duc d'Aiguillon et La Chalotais, Paris, Perrin, 1900-1901, à l'opposé des vues défendues par MARION, Marcel, La Bretagne et le duc d'Aiguillon..., op. cit. 
aucune mention de cette action, n'y attachant qu'une faible importance lorsque c'était le cas ${ }^{68}$. Les défenseurs du Guildo n'avaient en effet très probablement pas retardé les Britanniques dans leur tentative de rejoindre leurs navires pour la simple raison que tel n'était pas leur objectif. Si Bligh avait souhaité réembarquer, il aurait pu le faire le soir du 9 septembre, ou le 10, bien avant que d'Aiguillon soit en position de combattre. Pocquet savait aussi sans doute qu'aucun récit britannique - non plus que français - ne mentionnait Rioust, alors que celui-ci proclama que Bligh était venu en personne chez lui pour se venger. Mais ce manque de preuve confirmait simplement le message central des 3000 pages de l'œuvre que l'historien cosigne avec La Borderie : la Bretagne comme éternelle victime.

Dans ce cas, la condescendance française et la perfidie britannique auraient formé une sorte de conspiration du silence contre le véritable héros, le Léonidas breton...

L'analyse de la mémoire de Saint-Cast ici proposée n'est bien évidemment que très - trop - partielle. La mémoire strictement locale de l'événement reste encore à analyser avec minutie, au même titre que la mémoire globale, portée pour l'essentiel par une historiographie anglo-saxonne particulièrement prolixe sur la guerre de Sept Ans.

Les débats et les écrits entre érudits sur lesquels nous nous sommes focalisés ici n'en ont pas moins été importants dans la construction, au $\mathrm{XIX}^{\mathrm{e}}$ siècle, d'une certaine identité bretonne, en fournissant quelques-uns des éléments symboliques et matériels constitutifs d'une nation digne de ce nom : au-delà d'une langue, d'un folklore, d'un paysage typique ou d'une mentalité particulière, " une histoire [...], une série de héros parangons [...], des hauts lieux " comme a pu le noter A.-M. Thiesse ${ }^{69}$. Face à d'Aiguillon et à l'État français, Rioust, le Léonidas breton, héros autochtone,

68. Les mentions explicites à l'historiographie anglaise restent rares dans les publications de nature historique du temps; notons cependant les fréquentes références à Smolett à partir de la publication de sa relation dans OGÉE, Jean-Baptiste, Dictionnaire historique..., op. cit., p. 737-739 ou encore l'attention portée au récit de David Hume concernant l'opération contre Lorient en 1746 (CARRON, Jules, "Attaque des Anglais contre la ville de Lorient. Relation anglaise composée par David Hume ", Association bretonne, 1886, p. 144-168 et "Les Anglais en Bretagne : attaque des Anglais contre la ville de Lorient ", Revue de Bretagne et de Vendée, août 1887, p. 81-105).

69. ThiEsse, Anne-Marie, La Création des identités nationales. Europe, XVIII -XIXe siècles, Paris, Seuil, 2001, p. 14. L'on peut noter ici que plusieurs études récentes ont mis en évidence l'importance de la guerre de Sept Ans dans ce processus de création des identités nationales, tant en France (BELl, David, The Cult of the Nation in France. Inventing Nationalism, 1680-1800, Cambridge, Havard UP, 2001 ou DzIEMBowsKI, Edmond, Un nouveau patriotisme français [1750-1770]. La France face à la puissance anglaise à l'époque de la guerre de Sept Ans, Oxford, Voltaire Foundation, 1998) qu'à l'échelle des futurs États-Unis (ANDERSON, Fred, Crucible of War: The Seven Years' War and the Fate of Empire in British North America, 1754-1766, London, Faber, 2000 et The War that made America. A Short History of the French and Indian War, New York, Viking, 2005). Il convient alors de se demander si les phénomènes décrits ici pour la Bretagne correspondent à l'écho, à une moindre échelle, de ces phénomènes globaux ou si, au contraire, il ne s'agirait pas plutôt 
les Thermopyles du Guildo et la saga qui s'y serait jouée tout autant que la victoire à Saint-Cast des volontaires bretons, nobles et paysans, de Haute et de Basse-Bretagne permettaient de répondre au "besoin d'épopée " d'une nation en voie - selon certains - de (re)constitution ${ }^{70}$. Participant à sa manière de "l'invention de la Bretagne " - ou, plus exactement sans doute, d'une Bretagne ${ }^{71}$-, le monument de Saint-Cast serait alors venu matérialiser ce qui pouvait faire désormais figure de "mythe " national; il constituerait ainsi l'un de ces " lieux de mémoires " dominants, " spectaculaires et triomphants, imposants et généralement imposés, qu'ils le soient par une autorité nationale ou un corps constitué, mais toujours d'en haut, ont souvent la froideur ou la solennité des cérémonies officielles ", de ces lieux où "l'on se rend plus qu'on [ne] va ${ }^{72}$ ".

À ce titre, il n'est pas anodin de remarquer que Saint-Cast reste non seulement l'un des rares " lieux de mémoire " bretons de ce type, aux côtés de ceux de Sainte-Anne d'Auray et de la Pointe Saint-Mathieu pour la Grande Guerre, de celui de Saint-Aubin-du-Cormier aussi ${ }^{73}$, mais encore l'un des tout premiers du genre : édifié en 1858, le monument avait été envisagé dès les années $1820^{74}$. Il s'agit surtout de l'un des seuls - le seul -, à se départir d'un " modèle mémoriel breton " dominé par la déploration et la victimisation.

d'une réaction à ces phénomènes : le développement d'un " patriotisme " français aurait alors induit le renforcement d'une identité bretonne distincte.

70. C'est cette idée que met en exergue le titre de l'ouvrage assez récent de LA ConDAMINE, Pierre de, L'épopée de la Bretagne..., op. cit. Le chapitre du livre consacré aux affrontements du Guildo est intitulé "Ce furent des instants d'épopée ".

71. Nous reprenons ici l'expression de BERTHO, Catherine, "L'invention de la Bretagne. Genèse sociale d'un stéréotype ", Actes de la recherche en sciences sociales, 1980, $\mathrm{n}^{\circ} 35$, p. 45-62, appliquée à une réalité en partie différente.

72. NORA, Pierre, "Entre mémoire et histoire. La problématique des lieux ", dans NorA, Pierre (dir.), Les Lieux de mémoire, Paris, Gallimard, 1997, p. 42. Il les distingue des « lieux de mémoire " dominés, qui "sont les lieux refuges, le sanctuaire des fidélités spontanées et les pèlerinages du silence " constituant " le cour vivant de la mémoire ".

73. La chapelle votive érigée près d'Auray en l'honneur des émigrés tués à Quiberon en 1795 date de 1828; la mémoire qu'elle porte se révèle cependant à la fois moins strictement bretonne et beaucoup moins consensuelle, comme le laisse entendre la " réplique " bleue que constitue la statue de Hoche élevée à Quiberon en 1902.

74. L'on peut noter - qui s'en étonnera? - le caractère concomitant de ce mouvement à l'échelle de la Bretagne et de la France. 


\section{RÉSUMÉ}

La très modeste victoire de Saint-Cast, remportée le 11 septembre 1758 sur des troupes britanniques en cours de rembarquement, prend au cours du $\mathrm{XIX}^{\mathrm{e}}$ siècle dans l'historiographie bretonne une importance sans grand rapport avec l'impact réel de la bataille sur le cours de la guerre de Sept Ans. Historiens et érudits participent alors à la construction d'une sorte de " mythologie " régionale/nationale. Celle-ci s'articule autour de trois épisodes : l'appropriation bretonne de l'événement "Saint-Cast ", la disqualification du héros initial, allochtone, qu'est le duc d'Aiguillon, enfin l'effort en vue de la construction d'un nouveau héros, autochtone celui-là, Rioust des Villes-Audrain.

\section{ABSTRACT}

The victory won by the French army at Saint-Cast on the 11 September 1758 over a British expeditionary force was modest, but it took on a significance in Breton nineteenth-century historiography which had little relation to the real impact of the battle on the course of the Seven Years' War. Historians and local scholars joined forces to construct a kind of regional, one might even say 'national', mythology about the battle. This mythology developed around three episodes: the Breton appropriation of the Saint-Cast "event"; the disqualification of the initial hero, the Duke d'Aiguillon, considered an but "outsider"; and finally the creation of a new "insider" hero, Rioust des Villes-Audrain. 